\title{
Genomic and Phylogenetic Analysis of Zika Virus Isolates from Asymptomatic Blood Donors in the United States and Puerto Rico, 2016
}

\author{
Felipe Lopes Assis, Emilia Sippert, Bruno Coelho Rocha, Evgeniya Volkova, Rafaelle Fares-Gusmao, Suzan Ok, Caren Chancey, \\ and Maria Rios* \\ Division of Emerging and Transfusion Transmitted Diseases (DETTD), Office of Blood Research and Review (OBRR), Center for Biologics \\ Evaluation and Research (CBER), Food and Drug Administration (FDA), Silver Spring, Maryland
}

\begin{abstract}
Zika virus (ZIKV) caused a public health threat in the United States in 2016, leading to rapid development and implementation of blood screening assays for ZIKV RNA. Several ZIKV sequences from clinical cases have been reported, but none from asymptomatic/pre-symptomatic infections. We isolated and sequenced ZIKV from asymptomatic/presymptomatic blood donor (ABD-ZIKV) samples and compared with reported clinical sequences. Twelve ABD-ZIKV isolates were produced from 67 cultivated samples, and isolates were genetically similar among themselves. Most isolates shared mutations with the clinical isolate PRVABC59 2015, whereas two ABD-ZIKV isolates shared specific mutations with U.S. clinical isolates from 2016. The ABD-ZIKV strains clustered into two distinct subclades: one comprised mostly ABD-ZIKV from Puerto Rico, and another one comprised ABD-ZIKV from Florida and QTX-02 isolate (Puerto Rico). In this study, we showed the circulation of two slightly distinct virus strains among Puerto Rico blood donors, one of which was also reported in Florida.
\end{abstract}

Zika virus (ZIKV) is an emerging human-pathogenic Flavivirus, first discovered in 1947 in Uganda. ${ }^{1}$ Zika virus has become a public health concern since it started spreading from Asia through Pacific to the American continents. ${ }^{2}$ The first sizable ZIKV outbreak in humans occurred in 2007 on Yap Island, Federated States of Micronesia, which affected $73 \%$ of the population and was mostly associated with rash, arthralgia, and conjunctivitis. ${ }^{3}$ Spreading to other Pacific islands, ZIKV caused a large outbreak in French Polynesia in 2013/14. ${ }^{4}$ Zika virus was first detected in the Americas in 2014 on Easter Island. ${ }^{5}$ The first-reported vector-borne ZIKV transmission in the continental Americas occurred in October 2014 in Brazil, ${ }^{6}$ where more than 56,000 ZIKV infection cases were reported in 2015 , of which $5 \%$ approximately were associated with congenital syndrome and/or neurological complications. ${ }^{7}$ After its introduction into Brazil, ZIKV spread throughout South and Central America, reaching the Caribbean islands in mid-2015. ${ }^{8}$ Phylogenetic studies have identified two main ZIKV genotypes: the African and Asian genotypes, ${ }^{9}$ with the Asian genotype being responsible for the outbreaks in the Americas. ${ }^{8}$

During January 2015-February 2016, a total of 116 travelassociated Zika disease cases among residents of 33 U.S. states and the District of Columbia were reported. ${ }^{10}$ The first local mosquito-borne transmissions of ZIKV in a U.S. territory were reported in Puerto Rico in December 2015, and in the contiguous United States in July and November 2016, in Florida and Texas, respectively. ${ }^{11,12}$ Zika virus disease became a U.S. notifiable condition in 2016, and as of June 5 , 2019 , more than 43,110 cases of ZIKV disease had been reported in the United States and its territories (https:// www.cdc.gov/zika/reporting/). Most ZIKV infections ( 80\%) are asymptomatic, ${ }^{3}$ allowing infected people to be considered eligible to donate blood, which may result in viral transfusion

\footnotetext{
* Address correspondence to Maria Rios, Division of Emerging and Transfusion Transmitted Diseases (DETTD), Office of Blood Research and Review (OBRR), Center for Biologics Evaluation and Research (CBER), Food and Drug Administration (FDA), 10903 New Hampshire Ave., Bldg. 52/72, Rm. 5308, Silver Spring, MD 20993. E-mail: maria.rios@fda.hhs.gov
}

transmission, as reported in the Brazilian outbreak. ${ }^{13,14}$ This concern led the U.S. Food and Drug Administration (FDA) to recommend measures to minimize the risk of ZIKV transfusion transmission. ${ }^{15}$ Subsequently, the screening of the U.S. blood supply for ZIKV RNA was initiated using nucleic acid testing (NAT), under FDA-approved investigational studies. Bloodborne ZIKV transmission during asymptomatic or presymptomatic ZIKV infection is of concern for blood safety and the public health in general, mainly due to its impact on pregnancy and possibility of secondary exposure during sexual intercourse between transfusion recipients and their partners. Indeed, it has been reported that $45.3 \%(34 / 75)$ donors from Martinique who tested positive for ZIKV-RNA remained asymptomatic for at least 14 days post-donation. ${ }^{16}$

In this study, we report the isolation, complete genome sequencing, and phylogenetic analyses of 12 asymptomatic blood donor (ABD-ZIKV) isolates from 67 plasma specimens collected in 2016 from U.S. states $(n=8)$ and from Puerto Rico $(n=59)$. These samples were obtained from blood donations positive for ZIKV RNA by NAT screening and collected under Institutional Review Board (IRB)-approved informed consent provided by each of the institutions performing donor screening. The study and protocols for this research were reviewed and approved by the FDA Research Including Human Subjects Committee, Protocol \#17-001B. The FDA IRB-approved written informed consent was obtained from all blood donors involved in this study and retained by the collecting center.

The virus isolation was performed as described elsewhere. ${ }^{17}$ In brief, blood donor samples were cultivated in Vero cells or monocyte-derived macrophage (MDM) culture systems to rescue the virus, followed by virus expansion in cell cultures for up to three passages. Five of 12 (41.6\%) isolates were produced in Vero cell cultures, and the remaining seven isolates were produced only after cultivation in MDM cells, followed by virus expansion in cell lines.

Viral RNA was extracted using the QIAamp Viral RNA mini kit (Qiagen, Valencia, CA) and subjected to genome sequencing by Sanger after the amplification of 14 overlapping fragments with the One-step RT-PCR kit (Qiagen), according to the manufacturer's instructions (Supplemental Table S1). The 
5 ' - and 3'-terminal sequences were filled by genome cyclization followed by sequencing with specific primers (10492F and 232R), using the FirstChoice ${ }^{\mathrm{TM}}$ RLM-RACE $^{\mathrm{TM}}$ Kit (Invitrogen, Carlsbad, CA) and RNA 5' pyrophosphohydrolase enzyme (New England Biolabs Inc., Ipswich, MA).

Sequencing data analysis and genome assembling were performed using Sequencher 5.2.4 software (Gene Codes Corporation, Ann Arbor, MI). Complete genome sequences of the ABD-ZIKV isolates were produced and submitted to GenBank (Table 1). Natural selection codon-by-codon and phylogenetic analyses were performed in MEGA 7.0 software $^{18}$ (Pennsylvania State University, University Park, PA) using the maximum likelihood method (model of substitution: Tamura$\mathrm{Nei}$, including discrete gamma distribution with 5 rate categories and bootstrap of 1,000 replicates).

Genome sequences from 12 studied ABD-ZIKV showed high similarity with the prototype ZIKV strain PRVABC59 (KX377337.1), which was isolated in Puerto Rico in 2015 from a clinical case. Isolates from Puerto Rico had on average $99.75 \%$ similarity among them, except for QTX02-16, which had 99.95\% similarity with QTX04-16 from Florida, and 99.44\% average similarity when compared with other ABD-ZIKV isolates.

The ABD-ZIKV isolates showed mutations throughout their genomes when compared with PRVABC59 and other ZIKV strains (Supplemental Tables S2 and S3), and 45\% of these mutations were observed in NS5 and NS3. In addition, 20 common mutations were observed in QTX02-16 and QTX0416 , but not identified in the other 10 ABD-ZIKV isolates.

No mutations were observed in the $5^{\prime}$ region of $A B D-Z I K V$ isolates. By contrast, the $3^{\prime}$ region of each isolate showed a single mutation (CTS36-16p, CTS50-16p, CTS 56-16p, and CTS193-16p), multiple mutations (CTS30-16p, CTS47-16p, CTS183-16p, and QTX02-16), or no mutations (CTS61-16p, CTS178-16p, CTS223-16p, and QTX04-16) when compared individually with the PRVABC59 strain. In addition, the mutations observed in the ABD-ZIKV genomes were not under positive selection $(P$-value $<0.05)$ in a maximum likelihood analysis of natural selection codon-by-codon.

To investigate the origin of ABD-ZIKV isolates, we performed a phylogenetic analysis including a total of 446 ZIKV open reading frame (ORF) sequences available at GenBank. This dataset comprised complete nonredundant ORF sequences from both African and Asian clades, spanning the period from ZIKV discovery until its recent outbreak in the

TABLE 1

Blood donor-derived Zika virus isolates

\begin{tabular}{|c|c|c|c|c|}
\hline No. & Isolate ID & Origin & Year of collection & GenBank no. \\
\hline 1 & CTS30-16p* & Puerto Rico & 2016 & MH675619 \\
\hline 2 & CTS36-16p† & Puerto Rico & 2016 & MH675620 \\
\hline 3 & CTS47-16p† & Puerto Rico & 2016 & MH675621 \\
\hline 4 & CTS50-16p† & Puerto Rico & 2016 & MH675622 \\
\hline 5 & CTS56-16p‡ & Puerto Rico & 2016 & MH675623 \\
\hline 6 & CTS61-16p‡ & Puerto Rico & 2016 & MH675624 \\
\hline 7 & CTS178-16p‡ & Puerto Rico & 2016 & MH675625 \\
\hline 8 & CTS183-16p† & Puerto Rico & 2016 & MH675626 \\
\hline 9 & CTS193-16p‡ & Puerto Rico & 2016 & MH675627 \\
\hline 10 & CTS223-16p* & Puerto Rico & 2016 & MH675628 \\
\hline 11 & QTX02-16† & Puerto Rico & 2016 & MH675629 \\
\hline 12 & QTX 04-16* & Florida & 2016 & MH675630 \\
\hline
\end{tabular}

Americas in 2017. Our results were consistent with results of previous studies, showing the clustering of ZIKV strains into two main clades: African and Asian genotypes (Figure 1). Moreover, a ZIKV American lineage appears to have emerged from French Polynesian ZIKV strains (Asian genotype), which has Brazilian isolates as root for each clade. The American ZIKV isolates showed a geographical sub-clustering pattern grouping such isolates into four clades, including clade 1: Caribbean isolates (Haiti/2015 and Dominican Republic/ 2016), in addition to travel-related ZIKV isolates from the United States/2016, Russia/2016, Italy/2016, South Korea/ 2016, and the United Kingdom/2016; clade 2: South American isolates from Colombia 2015/2016, Venezuela/2016, and Peru/2016, as well as Panama/2015, Mexico/2016, and some travel-related isolates from Italy, Martinique, and United States; clade 3: Puerto Rico isolates, including the ABD-ZIKV isolates, except QTX02-16, in addition to isolates from China/ 2016, Suriname/2016, United States/2016, and Colombia/ 2016; and clade 4: ABD-ZIKV QTX02-16 and QTX04-16 isolates, besides isolates from Honduras/2016, Guatemala/ 2015, Nicaragua/2016, Mexico/2016, and travel-related isolates from United States/2016, China/2016, Singapore/2017, and Cuba/2017.

The genomic analysis (Supplemental Tables S2 and S3) showed only a few synonymous and non-synonymous mutations among ABD-ZIKV isolates, which corroborates previous studies showing a low within-outbreak substitution rate during Flavivirus circulation. ${ }^{2,19}$

Although the ZIKV isolate QTX02-16 was isolated from a Puerto Rico blood donor, it showed a genetic resemblance to the Florida isolate QTX04-16. Multiple introductions of ZIKV were necessary to give rise to the outbreak in Florida in 2016, ${ }^{20}$ with the Caribbean countries, including Puerto Rico, as the main source of ZIKV introduction into Florida by infected travelers coming or returning from areas experiencing ZIKV outbreaks. Our analysis suggests the circulation of two slightly distinct virus strains among Puerto Rico blood donors, one of which was also reported in Florida.

Our phylogenetic analysis showed the clustering of ABDZIKV isolates into two distinct subclades: 1) ZIKV isolates from Puerto Rico, except QTX02-16 isolate, branching with ZIKV strains from Puerto Rico and Colombia; and 2) QTX02-16 and QTX04-16 branching with ZIKV strains from Honduras, Nicaragua, Mexico, and the U.S. Caribbean countries, which have been described as the main source of ZIKV circulating in the United States. ${ }^{20}$

Our study reports the isolation and complete genome sequencing of ZIKV from ABD samples collected at the time of donation, which tested ZIKV RNA-reactive by NAT screening assays. The absence of post-donation follow-up information in this study limits our assessment of the outcome of infection, that is, if symptoms appeared later after blood donation. Nevertheless, the isolation of viable virus particles from blood units corroborates studies reporting cases of probable transfusion-transmitted ZIKV infection, which raises concerns about safety of blood transfusion mainly in endemic areas and during outbreaks.

The ABD-ZIKV isolates are genetically related to clinical ZIKV isolates from recent outbreaks in the Americas. Because no specific viral mutation was associated with asymptomatic infection, host genetic factors driving the ZIKV infection outcomes should be further investigated. Nevertheless, genetic 

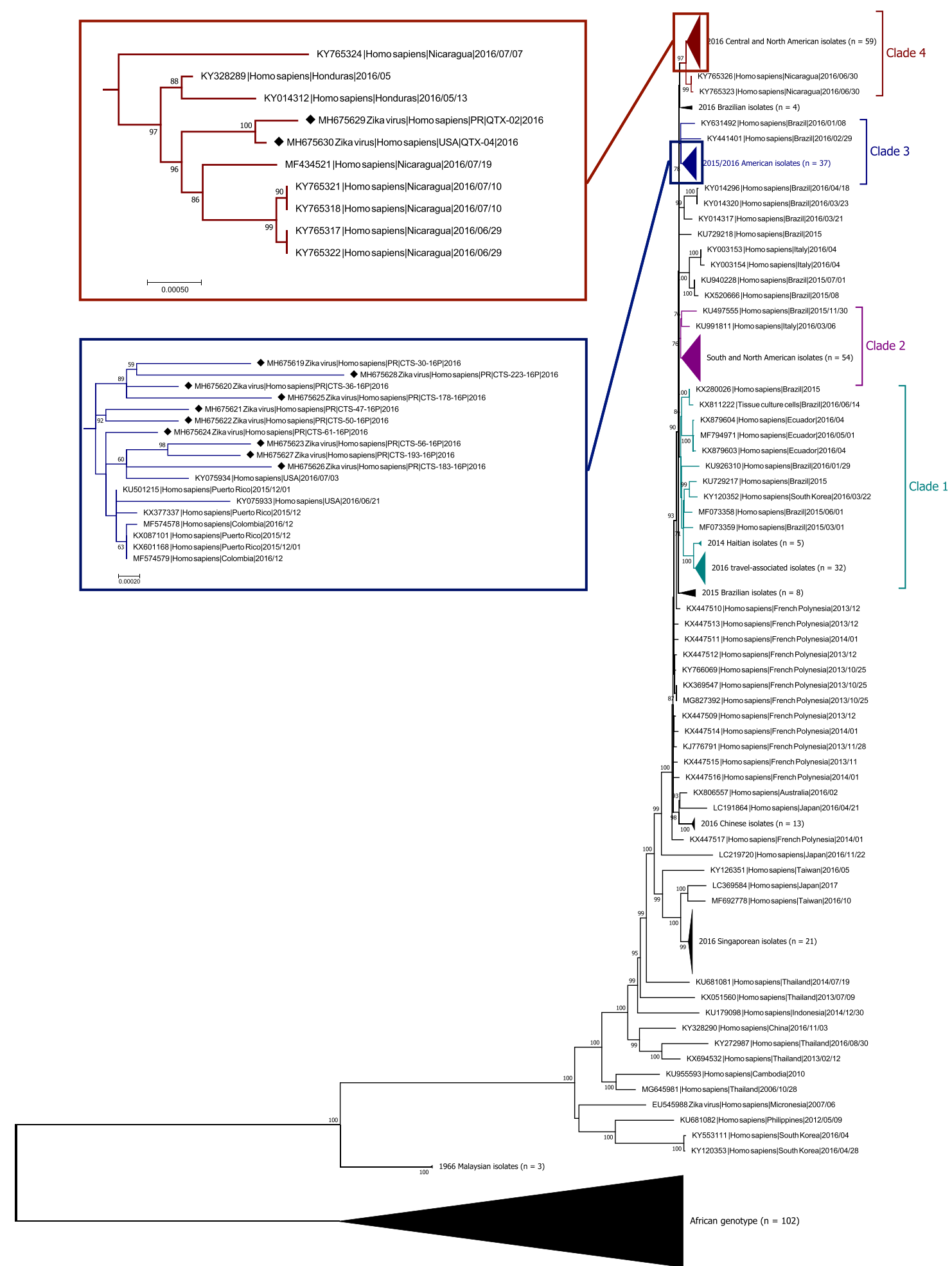

KX447510||Homo sapiens F French Polynesia|2013/12

KX447513|Homo sapiens|French Polynesial2013/12

kX447511|Homo sapiens|French Polynesia|2014/01

|X X47512||Homosapiens|French Polynesial2013/12

KY766069|Homo sapiens|French Polynesia|2013/10/25

KX369547|Homo sapiens|French Polynesia|2013/10/25

MG827392 |Homo sapiens|French Polynesial 2013/10/25

KX447509|Homo sapiens|French Polynesial2013/12

KX447514||Homos sapiens|French Polynesia|2014/01

KJ776791 HHomosapiens|French Polynesia|2013/11/2

KX447515|Homo sapiens||rench Polynesia|2013/11

KX447516|[Homo sapiens|French Polynesia|2014/0

KX806557/Homo sapiens/Australial2016/102

2016 Chinese isolates $(n=13)$. 2016/104/21

- LC219720/Homosapien/10pa 2014/1122

KY126351|Homosapiens|Taiwan|2016/05

L KY 26351 Homosapiens| Taivan|2016/05

L MF692778|Homosapiens|Taiwan|2016/10

100

Singaporean isolates $(n=21)$

KU681081|Homosapiens|Thailand|2014/07/19

KX051560|Homosapiens|Thailand|2013/07/09

KU179098|Homo sapienss|lndonesial2014/12/30

KY328290|Homosapiens|China|2016/11/1/03

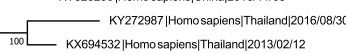

45981|Homo sapiens|Thailand|2006/10/28

EU545988ZZikavirus|Homosapiens|Micronesia|2007/0

KU681082|Homosapiens|Philippines|2012/05/09

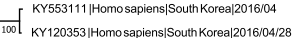

African genotype $(n=102)$

FIGURE 1. Phylogenetic analyses. Nonredundant complete open reading frame sequences from $446 \mathrm{ZIKV}$ isolates retrieved at ViPR database, and the 12 complete ZIKV sequences obtained from the United States. Blood donor-derived ZIKV isolates were included in this analysis. The analysis was performed using MEGA 7.0 software (maximum likelihood method and 1,000 bootstrap replicates). Branches containing ZIKV blood donor isolates were expanded, and diamonds indicate the isolate's position in the branches. The branch lengths were measured in the number of substitutions per site (scale length of 0.01). Bootstraps values lower than 70 and 55 were hidden in the tree and expanded branches, respectively. The cutoff value of $50 \%$ was used to calculate the condensed tree. Overrepresented branches were compressed, and the number of sequences ( $n$ ) of each group is given within brackets. This figure appears in color at www.ajtmh.org. 
studies of ZIKV isolates are required to monitor the ZIKV evolution and changes of viral pathogenesis during outbreaks, and to assess the effectiveness of available commercial donor screening and diagnostic assays for detection of genetic variants that may appear in future outbreaks.

Received October 8, 2019. Accepted for publication December 7, 2019.

Published online February 10, 2020.

Note: Supplemental tables appear at www.ajtmh.org.

Acknowledgments: We are grateful to the test developers and blood banks for providing the samples used in this study. This project was funded by the FDA Medical Countermeasures Initiative (MCMi), Grant OCET 2016-331, and supported in part by an appointment to the ORISE Research Fellowship Program at the Center for Biologics Evaluation and Research administered by the Oak Ridge Institute for Science and Education through an interagency agreement between the U.S. Department of Energy and the U.S. Food and Drug Administration.

Authors' addresses: Felipe Lopes Assis, Emilia Sippert, Bruno Coelho Rocha, Evgeniya Volkova, Rafaelle Fares-Gusmao, Suzan Ok, Caren Chancey, and Maria Rios, Division of Emerging and Transfusion Transmitted Diseases (DETTD), Office of Blood Research and Review (OBRR), Center for Biologics Evaluation and Research (CBER), Food and Drug Administration (FDA), Silver Spring, MD, E-mails: felipe.assis@fda.hhs.gov, emilia.sippert@fda. hhs.gov, brunocr2@gmail.com, evgeniya.volkova@fda.hhs.gov, rafaelle. gusmao@fda.hhs.gov, suzan.ok@gmail.com, caren.chancey@fda.hhs. gov, and maria.rios@fda.hhs.gov.

\section{REFERENCES}

1. Dick GW, Kitchen SF, Haddow AJ, 1952. Zika virus. I. Isolations and serological specificity. Trans $R$ Soc Trop Med Hyg 46: 509-520.

2. Metsky HC, 2017. Zika virus evolution and spread in the Americas. Nature 546: 411-415.

3. Duffy MR et al., 2009. Zika virus outbreak on Yap island, Federated States of Micronesia. N Engl J Med 360: 2536-2543.

4. Cao-Lormeau VM, Roche C, Teissier A, Robin E, Berry AL, Mallet HP, Sall AA, Musso D, 2014. Zika virus, French Polynesia, South Pacific, 2013. Emerg Infect Dis 20: 1085-1086.

5. Musso D, 2015. Zika virus transmission from French Polynesia to Brazil. Emerg Infect Dis 21: 1887.
6. Zanluca C, Melo VC, Mosimann AL, Santos GI, Santos CN, Luz K, 2015. First report of autochthonous transmission of Zika virus in Brazil. Mem Inst Oswaldo Cruz 110: 569-572.

7. Teixeira MG, Costa Mda C, de Oliveira WK, Nunes ML, Rodrigues LC, 2016. The epidemic of Zika virus-related microcephaly in Brazil: detection, control, etiology, and future scenarios. Am J Public Health 106: 601-605.

8. Faria NR et al., 2016. Zika virus in the Americas: early epidemiological and genetic findings. Science 352: 345-349.

9. Gong Z, Gao Y, Han GZ, 2016. Zika virus: two or three lineages? Trends Microbiol 24: 521-522.

10. Armstrong $P$ et al., 2016. Travel-associated Zika virus disease cases among U.S. residents-United States, January 2015February 2016. MMWR Morb Mortal Wkly Rep 65: 286-289.

11. CDC, 2019. Zika Cases in the United States. Available at: https:// www.cdc.gov/zika/reporting/2019-case-counts.html. Accessed October 3, 2019.

12. Shen S, Shi J, Wang J, Tang S, Wang H, Hu Z, Deng F, 2016. Phylogenetic analysis revealed the central roles of two African countries in the evolution and worldwide spread of Zika virus. Virol Sin 31: 118-130.

13. U.S. Department of Health and Human Services, Food and Drug Administration, Center for Biologics Evaluation and Research, 2018. Revised Recommendations for Reducing the Risk of Zika Virus Transmission by Blood and Blood Components (Intemet). Available at: https://www.fda.gov/downloads/BiologicsBloodVaccines/ GuidanceComplianceRegulatoryInformation/Guidances/Blood/ UCM518213.pdf. Accessed October 3, 2019.

14. Barjas-Castro ML et al., 2016. Probable transfusion-transmitted Zika virus in Brazil. Transfusion 56: 1684-1688.

15. Motta IJ et al., 2016. Evidence for transmission of Zika virus by platelet transfusion. N Engl J Med 375: 1101-1103.

16. Gallian P, Cabie A, Richard P, Paturel L, Charrel RN, Pastorino B, Leparc-Goffart I, Tiberghien P, de Lamballerie X, 2017. Zika virus in asymptomatic blood donors in Martinique. Blood 129: 263-266.

17. Sippert E, Rocha BC, Assis FL, Ok S, Rios M, 2019. Use of monocyte-derived macrophage culture increases Zika virus isolation rate from human plasma. Viruses 11: E1058.

18. Kumar S, Stecher G, Tamura K, 2016. MEGA7: molecular evolutionary genetics analysis version 7.0 for bigger datasets. Mol Biol Evol 33: 1870-1874.

19. Sall AA, Faye O, Diallo M, Firth C, Kitchen A, Holmes EC, 2010. Yellow fever virus exhibits slower evolutionary dynamics than dengue virus. J Virol 84: 765-772.

20. Grubaugh ND et al., 2017. Genomic epidemiology reveals multiple introductions of Zika virus into the United States. Nature 546: 401-405. 